\title{
First radius measurements of very low mass stars with the VLTI ${ }^{\star}$
}

\author{
D. Ségransan ${ }^{1}$, P. Kervella ${ }^{2}$, T. Forveille ${ }^{3,4}$, and D. Queloz ${ }^{1}$ \\ 1 Observatoire de Genève, 51 chemin des maillettes, 1290 Sauverny, Switzerland \\ 2 European Southern Observatory, Casilla 19001, Vitacura, Santiago 19, Chile \\ 3 Observatoire de Grenoble, BP 53X, 38041 Grenoble Cedex, France \\ ${ }^{4}$ Canada-France-Hawaii Telescope Corporation, PO Box 1597, Kamuela, HI 96743, USA
}

Received 15 October 2002 / Accepted 21 November 2002

\begin{abstract}
We present 4 very low mass stars radii measured with the VLTI using the $2.2 \mu \mathrm{m}$ VINCI test instrument. The observations were carried out during the commissioning of the 104-meter-baseline with two 8-meter-telescopes. We measure angular diameters of 0.7-1.5 mas with accuracies of 0.04-0.11 mas, and for spectral type ranging from M0V to M5.5V. We determine an empirical mass-radius relation for $\mathrm{M}$ dwarfs based on all available radius measurements. The observed relation agrees well with theoretical models at the present accuracy level, with possible discrepancy around $0.5-0.8 M_{\odot}$ that needs to be confirmed. In the near future, dozens of M dwarfs radii will be measured with $0.1-1 \%$ accuracy, with the VLTI, thanks to the improvements expected from the near infrared instrument AMBER. This will bring strong observational constraints on both atmosphere and interior physics.
\end{abstract}

Key words. stars: low-mass, brown dwarfs - stars: fundamental parameters - techniques: interferometric

\section{Introduction}

Mass, radius, luminosity and chemical composition are the basic physical properties of a star. For a given mass and chemical composition, theory can predict most of the stellar physical parameters at a given age. Accurate stellar mass, radius and luminosity measurements thus provide a crucial test of our understanding of stellar physics. To be relevant, these parameters must be determined with an accuracy of the order of 1\% (Andersen 1991). The previously rather noisy MassLuminosity relation for $\mathrm{M}$ dwarfs has recently been greatly improved (Delfosse et al. 2000; Ségransan et al. 2003). By contrast, the empirical Mass-Radius relation remains poorly constrained, since it is based on the observations of just two of the three known M-type eclipsing binaries, plus three $\mathrm{M}$ dwarfs radii measured at the Palomar Testbed Interferometer (PTI) with accuracies of $2-4 \%$ (Lane et al. 2001).

In this paper we present direct angular diameter measurements of four $\mathrm{M}$ dwarfs with spectral types ranging from M0V to M5.5V obtained with the Very Large Telescope Interferometer (VLTI) and two 8-meter-telescopes (Glindemann et al. 2001). The first two sections describe the observations and the data reduction. The third section presents the visibility calibration and the angular diameter determination. In the last section we compare presently known $\mathrm{M}$ dwarfs radiis and masses to theoretical models.

Send offprint requests to: D. Ségransan,

e-mail: Damien.Segransan@obs.unige.ch

* Based on observations made with the European Southern Observatory telescopes and obtained from the ESO/ST-ECF Science Archive Facility.

\section{Observations}

G1887, G1205 and Gl191 were observed during the first commissioning run of the VLTI with 8-meter-telescopes in early November 2001, on the second and third nights after the "first fringes" with these telescopes. The observations of G1551 were obtained in February 2002.

VINCI is the commissioning instrument of the VLTI (Kervella et al. 2000). It uses single-mode optical fibers to recombine the light from two telescopes of the Paranal Observatory, and modulates the optical path difference around its zero position to produce interference fringes. This recombination scheme was first used in the FLUOR instrument (Coudé du Foresto et al. 1998) and produces high precision visibility values, thanks to the efficient spatial filtering of the incoming beams and to the photometric monitoring of the filtered wavefronts.

Transfer function calibrators (Table 1) were selected based on their spectral type, distance to the target and known diameter (Cohen et al. 1999). Calibration tests show that a $\pm 1 \sigma$ shift in the calibrator angular diameter values modify the measured $\mathrm{M}$ dwarf radii by less than $0.8 \sigma$.

The $\mathrm{M}$ dwarfs science targets and their calibrators were observed in series of a few hundred interferograms, recorded at a frequency of 500-700 Hz. Due to many down times associated with the technical commissioning of the array, the observations of calibrator stars were not possible to the extent usually required to monitor extensively the transfer function. Nevertheless, the calibration measurements proved sufficient to estimate the transfer function with a good accuracy. The global interferometric efficiency of the VLTI mostly depends on two 
Table 1. Calibrators and instrumental+atmospherical transfer function. Calibrator uniform disk angular diameters come from Cohen et al. (1999). Internal error and external error on the transfer function are given for each calibrator. Fomalhaut angular diameter was redetermined using the VLTI. $(*)$ calibrator was not used due low visibilities or poor accuracy on their diameters.

\begin{tabular}{lccc|ccc}
\hline \hline & & Spec. & DIAMETER & \multicolumn{3}{|c}{ TRANSFER FUNCTION } \\
Targets & Calib. & Type & $\theta_{\text {UD }}[$ mas $]$ & $\left|T_{\mathrm{a}+\mathrm{i}}\right|^{2}$ & $\sigma_{\text {tot }}$ & $\left(\sigma_{\text {stat }} / \sigma_{\text {cal }}\right)$ \\
\hline GJ205 & 39-Eri & K3III & $1.81 \pm 0.02$ & 0.478 & 0.005 & $(.002 / .005)$ \\
& HD36167* & K5III & $3.55 \pm 0.06$ & 0.486 & 0.037 & $(.006 / .036)$ \\
GJ887 & HR8685 & M0III & $2.01 \pm 0.02$ & 0.448 & 0.005 & $(.003 / .004)$ \\
& Fomalhaut* $^{*}$ & A3V & $2.13 \pm 0.06$ & 0.433 & 0.013 & $(.004 / .013)$ \\
GJ191 & 39-Eri & K3III & $1.81 \pm 0.02$ & 0.420 & 0.005 & $(.004 / .004)$ \\
& Gam02 Vol* & K0III & $2.45 \pm 0.06$ & 0.411 & 0.014 & $(.006 / .013)$ \\
GJ551 & HD110458 & K0III & $1.6 \pm 0.02$ & 0.509 & 0.006 & $(.004 / .004)$ \\
\hline
\end{tabular}

parameters: the atmospheric coherence time, which varies over timescales sometimes as short as a few minutes, and the instrumental efficiency which changes over timescales of a week. Our effective calibration rate oversamples the instrumental efficiency variations by orders of magnitude, but risks undersampling the atmospheric coherence time. Fortunately, the Paranal observatory site is heavily monitored, and we could rely on continuous measurement of the atmospherical coherence time (Sandrock et al. 2000) to discard data intervals when the coherence time changed significantly between the observation of the calibrators and science targets.

\section{From fringes to visibilities}

We used a customised version of the VLTI/VINCI data reduction pipeline, whose general principle is based on the original FLUOR algorithm (Coudé du Foresto et al. 1997). Despite the high modulation frequency of the fringes, many recorded interferograms present a differential piston signature, as well as strong photometric fluctuations. The latter are expected for large apertures without adaptive optics correction, as individual speckle come in and out of the fiber input aperture. To overcome the noise amplification caused by low photometry data points, which could strongly bias the resulting visibilities, interferograms were calibrated by the average value of the photometry over the fringe packet. The two calibrated output interferograms were then subtracted to remove residual photometric fluctuations (Kervella, in prep).

In parallel to the FLUOR algorithm, based on Fourier analysis, we implemented a time-frequency analysis (Ségransan et al. 1999) based on the continuous wavelet transform (Farge 1992). Instead of the projection onto a sine wave of the Fourier transform, the wavelet transform decomposes it onto a function, ie. the Wavelet, that is localised in both time and frequency. We use as a basis the Morlet Wavelet, a Gaussian enveloppe multiplied by sine wave. With the proper choice of the number of oscillations inside the Gaussian envelope, the Morlet wavelet closely matches a VINCI interferogram. It is therefore extremely efficient at localizing the signal in both time and frequency.

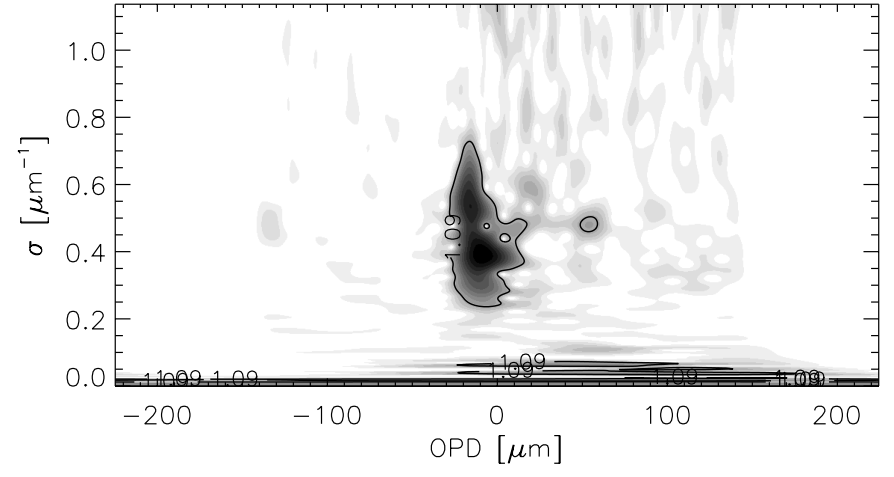

Fig. 1. Wavelet Spectral density of a pistonned interferogram. The effect of strong differential piston is to distort the interferogram fringe peak in the time-frequency space. Part of the interferogram's energy is spread out in both time and frequency, preventing a good measurement of the total energy.

As illustrated in Fig. 1, differential piston strongly affects the amplitude and the shape of the fringe peak in the wavelet power spectrum. We therefore select on the shape of fringe peak in the time-frequency domain to efficiently reject "pistonned" interferograms. We then derive visibilities from the wavelet spectral density, after removing the residual photon and detector noise.

\section{Angular diameter and limb darkening}

Stellar angular diameters are usually quoted for both a uniform disk and a limb-darkened disk, though only the latter is directly comparable to actual stellar models. The monochromatic visibility of a limb darkened disk is (Davis et al. 2000):

$V_{\lambda}=\frac{\int_{0}^{1} \mathrm{~d} \mu I(\mu) \mu J_{0}\left(\pi B \theta_{L D} / \lambda\left(1-\mu^{2}\right)^{1 / 2}\right)}{\int_{0}^{1} \mathrm{~d} \mu I(\mu)}$

where $I(\mu)$ is the surface brightness, $J_{0}$ is the zeroth order Bessel function, $B$ the projected baseline, $\lambda$ the wavelength, and $\theta_{\mathrm{LD}}$ the limb darkened diameter (in radian).

The VINCI instrument has no spectral resolution, and a bandpass defined by a $K^{\prime}$ filter $(2-2.3 \mu \mathrm{m})$. For marginally resolved disks $(B \theta / \lambda<<1)$, Eq. (1) remains approximately valid for polychromatic measurements, with $\lambda$ then being the effective wavelength of the system. It differs from the mean wavelength of the filter through the wavelength-dependency of fiber coupling, combiner transmission, and the stellar spectrum. Since interferograms are Fourier transforms of the instrumental bandpass, they provide, in principle, an accurate measurement of the effective wavelength, obtained as the average barycenter of the fringe peak in the spectral density space. We computed such effective wavelengths for each star and compared them to the one given by the VINCI instrument modelisation $\left(\lambda_{\mathrm{eff}}=2.195 \pm 0.010 \mu \mathrm{m}\right)$. Our estimations of $\lambda_{\mathrm{eff}}$ present a large scatter which is due to atmosperical differential piston and photometric fluctuations. We thus used the VLTI/VINCI effective wavelength provided by ESO and the effective temperature of each star to compute an accurate $\lambda_{\mathrm{eff}}$ for each target and calibrator. 


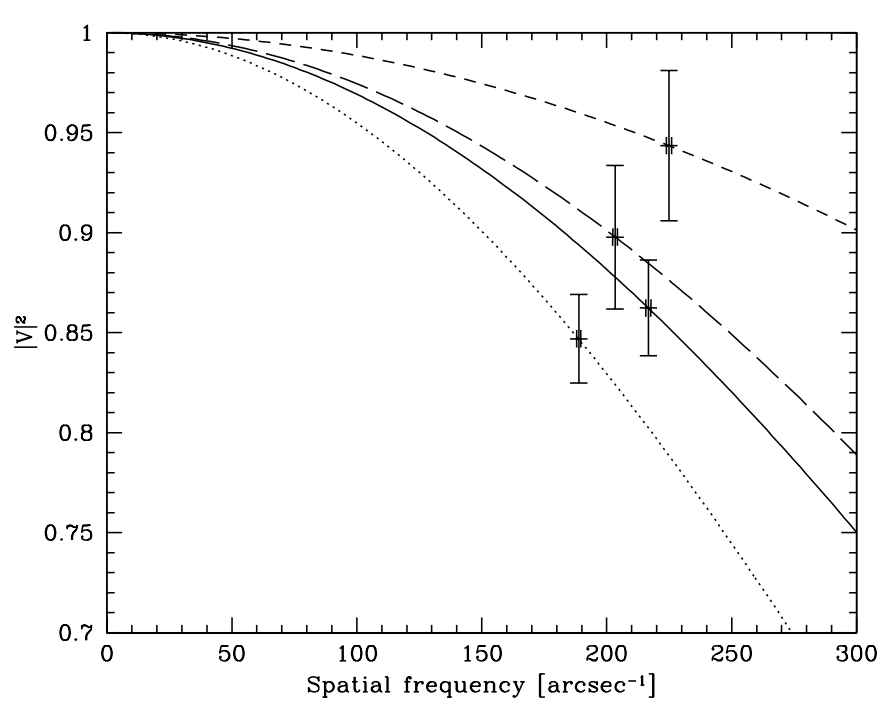

Fig. 2. Measured visibilities (data points) and best-fit model (curves, Eq. (2)) for GJ 205 (plain), GJ887 (dots), G1191 (short dash), and G1191 (long dash).

Due to their relatively short baselines, and limited uvcoverage, these observations cannot discriminate between an unphysical uniform star and a more realistic slightly larger limb-darkened star. To correct for limb-darkening, we therefore have to use a theoretical limb-darkening law. We adopt the nonlinear limb-darkening coefficients from Claret (2000), Eq. (2), based on atmospheric models from Allard et al. (1997). We use gravities and effective temperatures from Baraffe et al. (1998) isochrones at $5 \mathrm{Gyr}$ (Table 2) to select the apropriate entry in the Claret (2000) tables.

$I(\mu)=I(1)\left[1-\sum_{k=1}^{4} a_{k}\left(1-\mu^{k / 2}\right)\right]$.

In Eq. (2), the $a_{k}$ are the limb-darkening coefficients and $\mu=$ $\cos (\gamma)$, with $\gamma$ the angle between the line of sight and the emergent ray. The limb-darkening correction that we apply is in principle model-dependent. Fortunately, it is also quite small for $\mathrm{M}$ dwarfs, only 1-2\% (Table 2). The correction itself is at best marginally relevant at our current accuracy level for individual diameters, and its theoretical uncertainties can for now be safely neglected. Future observations with longer baselines, at shorter wavelengths, and with a more accurate calibration, will need to consider the issue more carefully, but they will also directly measure the limb-darkening law for the closest M dwarfs.

\section{Mass-radius relation}

An accurate empirical mass-radius relation is an essential constraint on stellar interior structure, evolutionnary models and atmospheric physics. The interior structure is largely determined by the equation of state, whose derivation for very low mass stars, brown dwarfs, and planets involves the complex physics of strongly correlated and partially degenerated quantum plasma (Chabrier \& Baraffe 2000).
To obtain the empirical mass-radius relation we need to convert angular diameter into linear radius, which is easily done thanks to accurate HIPPARCOS parallaxes for all sources, and we need accurate masses. For these single stars masses can only be estimated, from IR photometric measurements and accurate mass-luminosity relations. Fortunately, the $K$-band mass-luminosity relation has very little intrinsic dispersion for M dwarfs. We estimated the masses of the stars listed in Table 2 using an update (Ségransan et al. 2003) of the (Delfosse et al. 2000) empirical $K$ band mass-luminosity relation, now based on 27 accurate masses and luminosities. The data dispersion around the average empirical relation corresponds to a mass error of $\sim 5 \%$. Masses and radii are summarized in Table 2.

Figure 3 compares the empirical radii \& masses with 5 Gyr and 1 Gyr theoretical isochrones from Baraffe et al. (1998). The Baraffe et al. (1998) models reproduce the observation fairly well in the sampled range, between 0.65 and $0.12 M_{\odot}$. At a more detailed level though, one notices that the models underestimate the radii for YY Gem, V818 Tau and GJ 205, with masses in the $0.5-0.65 M_{\odot}$ range. The discrepancy is highly significant for the eclipsing binaries, as extensively discussed by Torres \& Ribas (2002) and is more marginal for long baseline interferometry data. There is an indication in Fig. 3 that the model reproduces the observations well below $0.5 M_{\odot}$, and only become discrepant above that value. If real, this suggests that the shortcomings of current models have to be searched in the energy transport (convection description, opacities), rather than in the equation of state (EOS). As their mass decreases, stars at the bottom of the main sequence have increasingly simple transport properties (they are fully convective below $\sim 0.3 M_{\odot}$ ), and an increasingly correlated and degenerate EOS. EOS shortcomings are therefore expected to show up most prominently at the lowest masses, and transport problems at higher masses. That result obviously needs confirmation from additional data points and from more accurate measurements, but the consistency between the eclipsing binary and the resolved single stars is comforting, as these two datasets were obtained through completely independent methods.

In addition to providing physical radii, the angular radii can be combined with integrated flux measurement to obtain the effective temperature from the Stefan-Boltzmann law. Up to now that process has typically been inversed, to derive radii for stars whose effective temperatures were obtained from comparison with model spectra (e.g. Leggett et al. 2000). Amongst the sources in Table 2, only GJ 699 has an integrated flux measurement (Leggett et al. 2000), and we thus have to rely on the bolometric correction polynomial fit of (Leggett et al. 2000) to estimate it for the other targets.

In spite of these increased uncertainties on the bolometric flux, the resulting effective temperatures have fairly small error bars (Table 2). With increasingly precise direct angular radii, and with integrated flux measurements for all targets, this test can become a very discriminating validation of model atmospheres, with potential accuracies at the $\sim 1 \%$ level on both $T_{\text {eff }}$ and $\log (g)$. The present diameter measurements, which have similar precisions to those obtained at PTI (Lane et al. 2001), have been obtained during the commissioning phase of 
Table 2. Measured angular diameter for the target stars. The uniform disk diameters for GJ 699, GJ 15A, GJ 411, GJ 380, GJ 105A are from Lane et al. (2001). For consistency with our measurements, we recomputed their limb darkened diameters, masses and radii. The masses for Gl 380 and Gl105A are based on the Baraffe et al. (1998) M-L relation, with an arbitrary 5\% error bar.

\begin{tabular}{|c|c|c|c|c|c|c|c|c|c|c|c|c|c|c|c|c|c|c|}
\hline \multirow[b]{2}{*}{ Object } & \multirow{2}{*}{$\begin{array}{c}\text { Spectral } \\
\text { Type }\end{array}$} & \multicolumn{2}{|c|}{ РНOTOMETRY \& MASSES } & \multicolumn{6}{|c|}{ LiMB DARKENING, $K$ BAND (models) } & \multicolumn{3}{|c|}{ DIAMETER [mas] } & \multicolumn{2}{|c|}{ RadIUs $\left[R_{\odot}\right]$} & \multicolumn{4}{|c|}{ ATM. PROP. } \\
\hline & & $M_{K}$ & $M / M_{\odot}$ & $g$ & $T_{\text {eff }}$ & $a_{1}$ & $a_{2}$ & $a_{3}$ & $a_{4}$ & $\theta_{\mathrm{UD}}$ & $\theta_{\mathrm{LD}}$ & $\sigma_{\theta}$ & $R$ & $\sigma_{R}$ & $T_{\text {eff }}$ & $\sigma_{T_{\mathrm{eff}}}$ & $g$ & $\sigma_{g}$ \\
\hline GJ205 & $\mathrm{M} 1.5 \mathrm{~V}$ & & $631+0031$ & 4.70 & 3894 & 1.11 & -1.11 & 0.92 & & 1.124 & 1.149 & 0.11 & 0.702 & 0.063 & 3520 & 170 & 1.54 & \\
\hline & & & & & 5 & & & & & & & & & & 26 & 56 & & \\
\hline & & & & 8 & 3419 & 76 & & 2.3 & & & 0.692 & 0.06 & 291 & 0.025 & 3570 & 156 & .96 & 0 . \\
\hline & $5.5 \mathrm{~V}$ & 80 & .006 & .19 & 3006 & 1.94 & -2.80 & 2.39 & -0.8 & 1.023 & 1.044 & 0.08 & 0.145 & 0.011 & 3042 & 117 & 5.20 & 0.23 \\
\hline & & & & & 319 & & & 2.54 & 8 & & 1.004 & 0.0 & 196 & 0.0 & & 65 & 55 & 0. \\
\hline & & & & & 3541 & 1.66 & & 2.14 & & & 1.000 & 0.05 & 383 & 0.0 & 3698 & 95 & 4.89 & 0.06 \\
\hline J411 & M1.5V & 33 & $0.403 \pm$ & 88 & 3533 & 1.67 & -2 & 2.16 & -0 . & 1.413 & 1.436 & 0.03 & 0.393 & 0.008 & 3570 & 42 & 4.85 & 0.03 \\
\hline & & & 67( & & 4106 & 1.09 & & 0.8 & & 1.268 & 1.13 & 0.04 & 0.605 & 0.0 & - & - & 4.70 & 0.03 \\
\hline GJ105A & $3 \mathrm{~V}$ & 17 & $0.790 \pm .039^{*}$ & .56 & 4603 & 0.86 & -0.53 & 0.38 & -0.13 & 0.914 & 0.936 & 0.07 & 0.708 & 0.05 & - & - & 4.63 & 0.05 \\
\hline
\end{tabular}

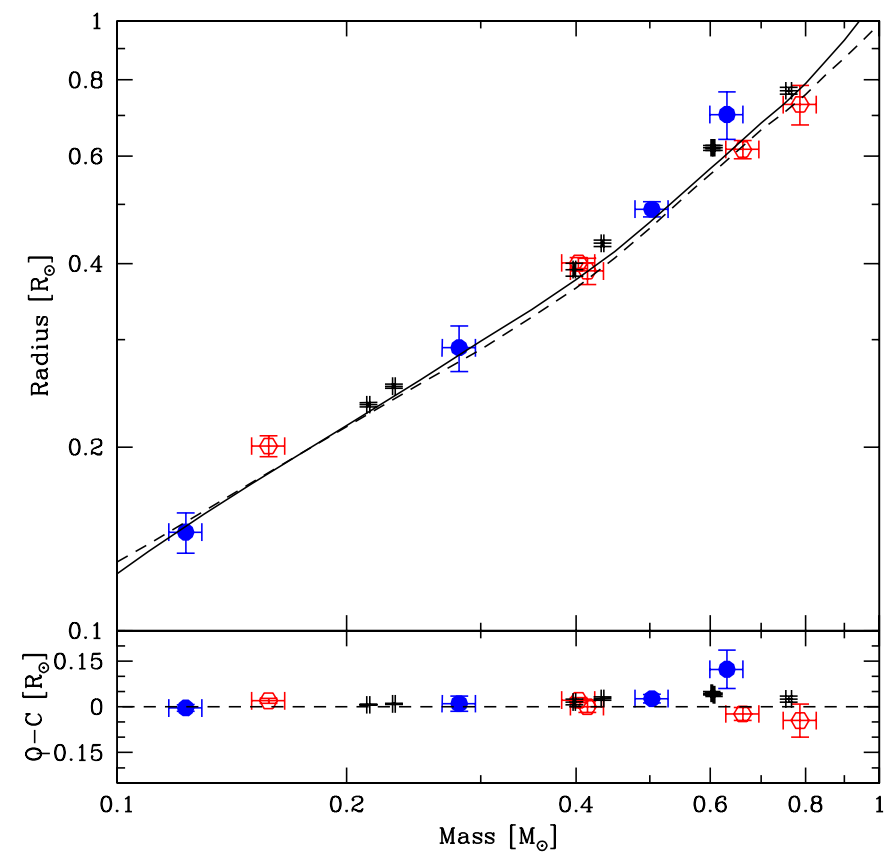

Fig. 3. Comparison between observational radii \& masses measurements and the theoretical mass-radius relation. The solid and dashed curves are the 5 Gyr and 0.4-1 Gyr theoretical isochrones of Baraffe et al. (1998), which do not differ much over the present mass range. The filled circles are radius measurements from this paper, the open circles are PTI measurements by Lane et al. (2001), and the dots are masses and radii of three eclipsing binaries (Metcalfe et al. 1996; Torres \& Ribas 2002; Ribas 2002). The error bars and the residuals from the model are shown at the bottom of the figure.

the VLTI interferometer, with a recombiner that was specified to validate and debug the interferometer, and not for an optimum scientific output. With the improvements expected from the near infrared scientific recombiner, AMBER (Petrov et al. 2001), from adaptive optics on the unit telescopes, the VLTI will measure dozens of $\mathrm{M}$ dwarfs with $0.1-1 \%$ accuracy. This will bring very strong observational constraints on models of both stellar interiors and atmospheres.
Acknowledgements. This research has made use of the SIMBAD database operated by CDS, Strasbourg, France. D.S. acknowledges the support of the Fonds National Suisse de la Recherche Scientifique. We thank the VLTI team for the commissioning and the data acquisition.

\section{References}

Allard, F., Hauschildt, P. H., Alexander, D. R., \& Starrfield, S. 1997, ARA\&A, 35, 137

Andersen, J. 1991, A\&A Rev., 3, 91

Baraffe, I., Chabrier, G., Allard, F., \& Hauschildt, P. H. 1998, A\&A, 337,403

Chabrier, G., \& Baraffe, I. 2000, ARA\&A, 38, 337

Claret, A. 2000, A\&A, 363, 1081

Cohen, M., Walker, R. G., Carter, B., et al. 1999, AJ, 117, 1864

Coudé du Foresto, V., Perrin, G., Ruilier, C., et al. 1998, in Astronomical Interferometry, Proc. SPIE, 3350, 856

Coudé du Foresto, V., Ridgway, S., \& Mariotti, J. 1997, A\&AS, 121, 379

Davis, J., Tango, W. J., \& Booth, A. J. 2000, MNRAS, 318, 387

Delfosse, X., Forveille, T., Ségransan, D., et al. 2000, A\&A, 364, 217

Farge, M. 1992, Annual Review of Fluid Mechanics, 24, 395

Glindemann, A., Ballester, P., Bauvir, B., et al. 2001, The Messenger, 106,1

Kervella, P., Coudé du Foresto, V., Glindemann, A., \& Hofmann, R. 2000, in Interferometry in Optical Astronomy, Proc. SPIE, 4006, 31

Lane, B. F., Boden, A. F., \& Kulkarni, S. R. 2001, ApJ, 551, L81

Leggett, S. K., Allard, F., Dahn, C., et al. 2000, ApJ, 535, 965

Metcalfe, T. S., Mathieu, R. D., Latham, D. W., \& Torres, G. 1996, ApJ, 456, 356

Petrov, R. G., Malbet, F., Weigelt, G., et al. 2001, in SF2A-2001: Semaine de l'Astrophysique Française, 615

Ribas, I. 2002, A\&A, accepted

Ségransan, D., Forveille, T., Millan-Gabet, C. P. R., \& Traub, W. A. 1999, in Working on the Fringe: Optical and IR Interferometry from Ground and Space, ASP Conf. Ser., 194, 290

Sandrock, S., Amestica, R., Duhoux, P., Navarrete, J., \& Sarazin, M. S. 2000, in Advanced Telescope and Instrumentation Control Software, Proc. SPIE, 4009, 338

Ségransan, D., Delfosse, X., Forveille, T., et al. 2003, in Brown Dwarfs, IAU Symp., 211, 211

Torres, G., \& Ribas, I. 2002, ApJ, 567, 1140 